\title{
A Rare Case of Retinal Artery Occlusion in the Context of Mediterranean Spotted Fever
}

\author{
Diana Beselga António Campos Miguel Castro Sílvia Mendes \\ Joana Campos Arminda Neves Luís Violante João Paulo Castro Sousa \\ Department of Ophthalmology, Hospital Center Leiria, Leiria, Portugal
}

\section{Key Words}

Mediterranean spotted fever $\cdot$ Retinal vasculitis $\cdot$ Branch retinal occlusion $\cdot$ Intravitreal triamcinolone

\begin{abstract}
Background: Mediterranean spotted fever is a zoonosis endemic in the Mediterranean region. The microorganism Rickettsia conorii is responsible for the disease due to its angiotropism for endothelial cells. It produces host cell necrosis, thrombosis, and organ dysfunction. Ophthalmologic manifestations are rare. Findings: The authors describe the case of a 55-year-old female with Mediterranean spotted fever who developed localized retinal vasculitis with associated macular edema. Treatment with intravitreal triamcinolone allowed a significant recovery of visual acuity. Conclusion: Ophthalmological symptoms in these patients should be emphasized because there can be severe ocular complications with a potentially irreversible loss of visual acuity.

(c) 2014 S. Karger AG, Basel
\end{abstract}

\section{Introduction}

Mediterranean spotted fever is a zoonosis endemic occurring predominantly in Mediterranean countries. The microorganism Rickettsia conorii, responsible for the disease, is an extremely fastidious Gram-negative organism which proliferates in the cytoplasm of eukaryotic cells. The disease is transmitted through a bite of a tick vector (arthropod Riphicephalus sanguineus) [1].

Angiotropism with multiplication in the host endothelial cells is the predominant feature leading to cell necrosis, thrombosis, and organ dysfunction. 
Beselga et al:: A Rare Case of Retinal Artery Occlusion in the Context of Mediterranean Spotted Fever

The classic diagnostic triad is characterized by fever, maculopapular rash (involving palms and soles), inoculation scars or tache noir.

The incubation period is between 5 and 7 days with a sudden onset in $50 \%$ of the cases. Disease duration is between 7 and 14 days. Fever may reach $40^{\circ} \mathrm{C}$, accompanied by headache, chills, myalgia, arthralgia, and anorexia. A maculopapular rash is usually characteristic and appears after the systemic symptoms [2].

Ophthalmologic manifestations described in the literature are rare and the most common findings are petechial lesions of the conjunctiva. Isolated case reports of oculoglandular Parinaud's syndrome, uveitis, optic neuropathy and retinal vasculitis have been reported [38].

Laboratory diagnostic methods may be used including isolation by culture, serological tests (indirect immunofluorescence), immunohistochemical detection in skin biopsies, and PCR amplification. The detection of specific antibodies (IgM and IgG) by indirect immunofluorescence is the method most commonly used. The demonstration of an IgM titer $\geq 1: 64$ and $\operatorname{IgG} \geq 1: 128$ and/or an increase to 4 times the size in the IgG titer is considered evidence of infection [2].

The treatment of choice is doxycycline, since it is effective and achieves good intracellular levels.

\section{Case Report}

A 55-year-old healthy Caucasian female with no history of ocular disease was observed in the emergency department with fever, myalgia, nasal congestion with 3 days of evolution, and a generalized rash for 1 day. She had contact with dogs. Physical examination revealed a cooperating and well-oriented patient with stable vital parameters and a tympanic temperature of $38.2^{\circ} \mathrm{C}$. There was a maculopapular rash on the upper limbs, abdomen, and the dorsal region. A tache noir, a small crust surrounded by a violet halo, was visible in the region of the left thigh. There were no meningeal signs and no cardiorespiratory or abdominal changes. Serology for $R$ conorii was positive with an IgM titer of 1/320. Autoimmune and thrombophilia studies were negative. Hypercholesterolemia and systemic hypertension were excluded. The patient was admitted to our department and therapy with oral doxycycline $100 \mathrm{mg}$ every $12 \mathrm{~h}$ was initiated. Oral doxycycline was given for 10 days.

On the first day of hospitalization, the patient reported decreased visual acuity (VA) of the right eye (RE). A CT scan was performed which was normal. No ophthalmologic examination was requested.

There was a systemic improvement and the patient was discharged after 6 days. Five days thereafter, the patient returned to the emergency room with complaints of worsening of RE VA. Observation revealed an RE VA of 20/100 and there was a superior temporal artery branch occlusion with perivascular sheathing in the ocular fundus as well as cotton wool spots and edema (fig. 1). The retinal edema affected the macular region (fig. 2). Campimetric examination revealed an inferior nasal scotoma, consistent with fundus lesions (fig. 3).

Intravitreal injection of triamcinolone acetonide $3.2 \mathrm{mg} / 0.08 \mathrm{ml}$ was given only once and showed significant improvement of the retinal edema and a recovery of RE VA to 20/25 after 1 month. The vascular sheathing and the occlusion persisted (fig. 4), and so did the corresponding scotoma in the visual field.

The total follow-up period is 1 year. No ocular side effects related to intravitreal triamcinolone were detected. 
Beselga et al.: A Rare Case of Retinal Artery Occlusion in the Context of Mediterranean Spotted Fever

\section{Discussion}

In Portugal, the incidence of Mediterranean spotted fever remains one of the highest among countries in the Mediterranean region.

The course of the disease is usually self-limited and benign. The prognosis varies, depending on the virulence of the bacterial strain and the host comorbidities. Early treatment also affects the prognosis significantly.

Ocular manifestations are rare and usually benign in association with this disease. They are often undervalued compared to systemic manifestations. Angiotropism makes retinal vessels a potential target, and retinal lesions can be sight-threatening.

We describe a rare case of Mediterranean spotted fever and retinal vasculitis with associated macular edema. The lesion was detected only 10 days after the onset of symptoms and the patient had already started systemic therapy with doxycycline at this time. The mechanism of retinal injury is mainly inflammation and exudative with the macular edema, causing decreased VA. Thus, treatment with intravitreal corticosteroids (triamcinolone acetonide) allowed the resolution of macular edema and visual recovery.

During the course of the disease, ocular symptoms should be evaluated because severe ocular complications can occur with a potentially irreversible loss of VA.

\section{Disclosure Statement}

The authors report no proprietary interest or financial support and do not have a conflict of interest.

\section{References}

1 Oliveira J, Côrte-Real R: Rickettsia infections in Portugal. Acta Med Port 1999;12:313-321.

12 Pinna A: Ocular manifestations of rickettsiosis. 1. Mediterranean spotted fever: laboratory analysis and case reports. Int J Med Sci 2009;6:126-127.

-3 Pinna A, Sotgiu M, Carta F, Zanetti S, Fadda G: Oculoglandular syndrome in Mediterranean spotted fever acquired through the eye. Br J Ophthalmol 1997;81:172.

4 Rizo AE, Muñiz O, Auladell C, Miranda ML: A case of bilateral uveitis secondary to Mediterranean spotted fever. Clin Infect Dis 1992;14:624-625.

5 Pinna A, Sechi LA, Serru A, Zanetti S, Fadda G, Carta F: Endogenous panuveitis in a patient with Rickettsia conorii infection. Acta Ophthalmol Scand 2000;78:608-609.

6 Khairallah M, Ladjimi A, Chakroun M, Messaoud R, Yahia SB, Zaouali S, et al: Posterior segment manifestations of Rickettsia conorii infection. Ophthalmology 2004;111:529-534.

7 Leone S, De Marco M, Ghirga P, Nicastri E, Lazzari R, Narciso P: Retinopathy in Rickettsia conorii infection: case report in an immunocompetent host. Infection 2008;36:384-386.

-8 Agahan AL, Torres J, Fuentes-Páez G, Martínez-Osorio H, Orduña A, Calonge M: Intraocular inflammation as the main manifestation of Rickettsia conorii infection. Clin Ophthalmol 2011;5:1401-1407. 
Case Reports in

Ophthalmology
Case Rep Ophthalmol 2014;5:22-27

DOI: $10.1159 / 000358248$

Beselga et al.: A Rare Case of Retinal Artery Occlusion in the Context of Mediterranean Spotted Fever

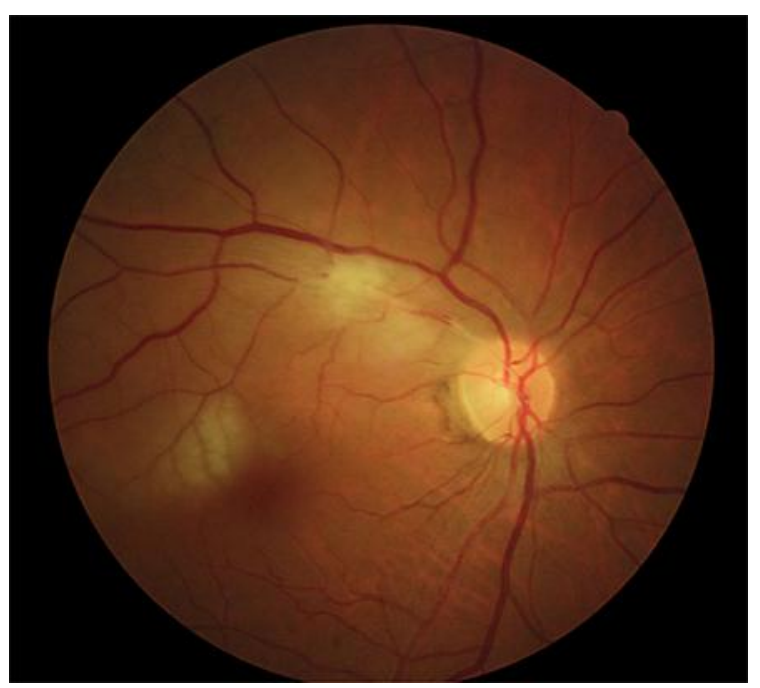

Fig. 1. Superior temporal artery branch occlusion with perivascular sheathing, cotton wool spots, and edema involving the macula.

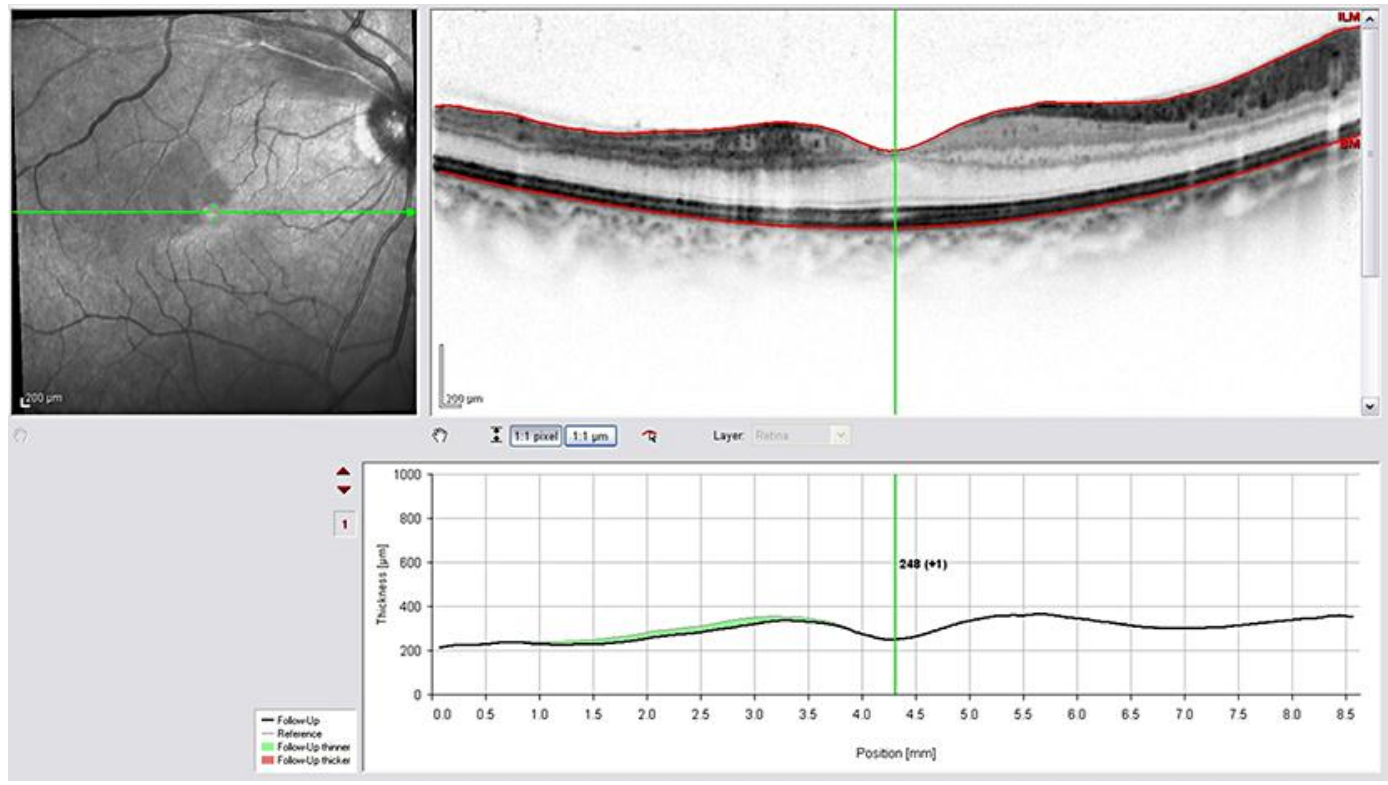

Fig. 2. OCT showing increased retinal thickness, mainly in the inner retina, consistent with nerve fiber layer edema. 


DOI: 10.1159/000358248

2014 S. Karger AG, Basel www.karger.com/cop

Beselga et al.: A Rare Case of Retinal Artery Occlusion in the Context of Mediterranean Spotted Fever

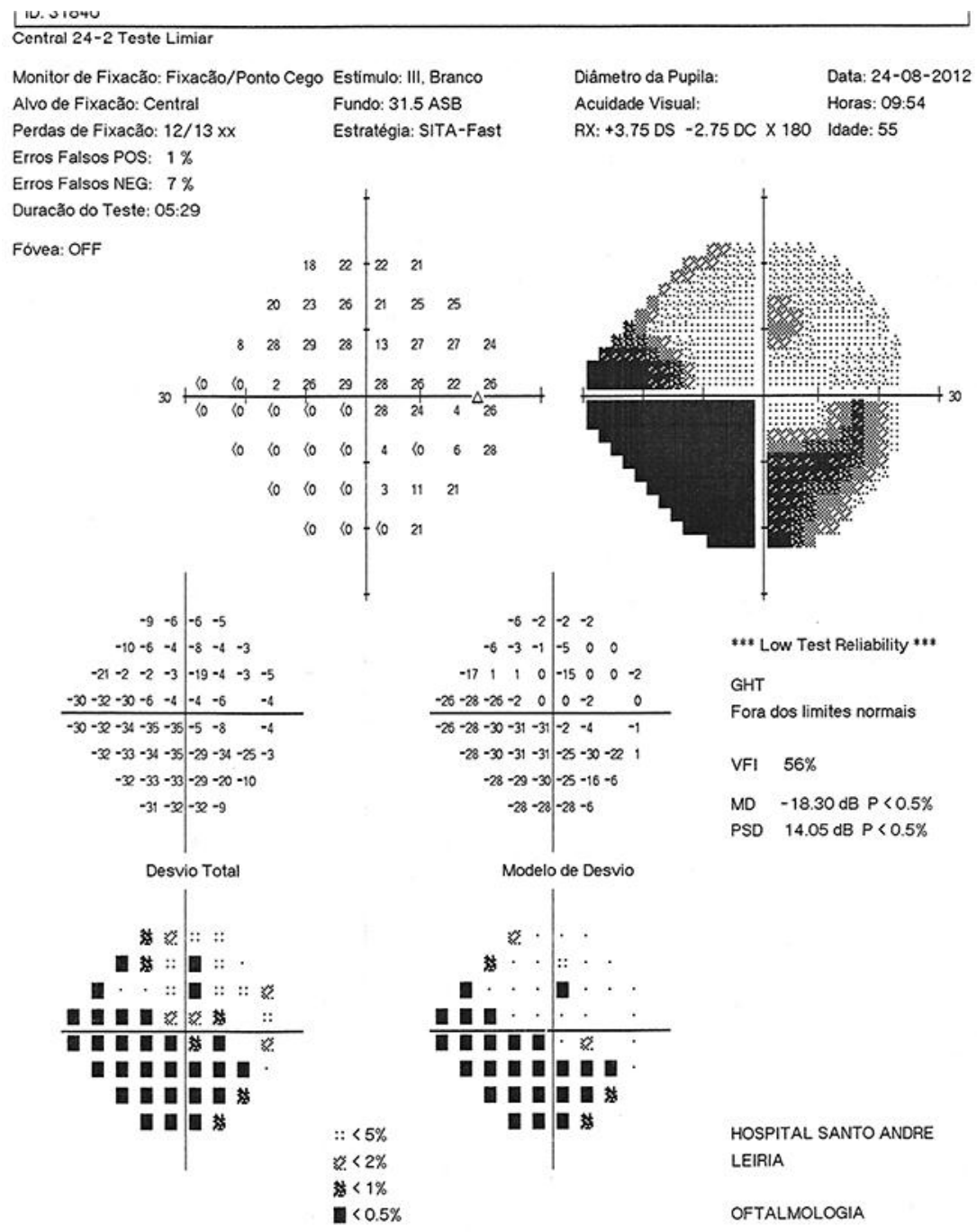

Fig. 3. Inferior nasal scotoma, consistent with fundus lesions. 
Case Reports in

Ophthalmology

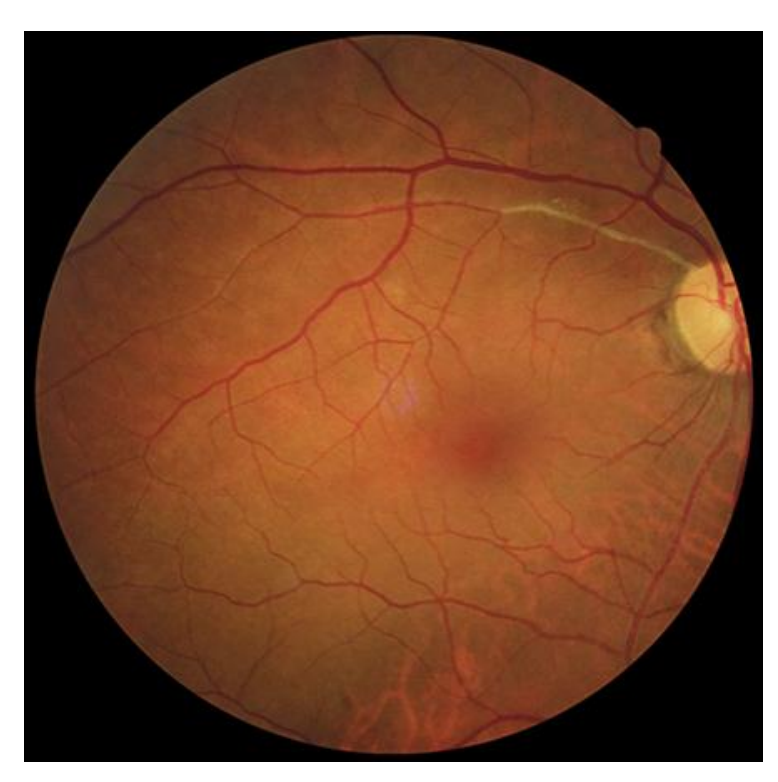

Fig. 4. Renal branch arterial sheathing and occlusion. \begin{tabular}{l|l}
\hline DOI: $10.1159 / 000358248$ & C 2014 S. Karger AG, Basel \\
\hline
\end{tabular}

www.karger.com/cop

Beselga et al:: A Rare Case of Retinal Artery Occlusion in the Context of Mediterranean Spotted Fever 